\title{
The association between Occlusion Time and Temporomandibular Disorders
}

\author{
Alberto Baldini*, Alessandro Nota, Paola Cozza \\ Department of Orthodontics, University of Rome Tor Vergata, Italy
}

\section{A R T I C L E I N F O}

\section{Article history:}

Received 18 September 2013

Received in revised form 19 August 2014

Accepted 20 August 2014

Available online $\mathrm{xxxx}$

\section{Keywords:}

Dental Occlusion

Craniomandibular disorders

Temporomandibular joint

Oral physiology

Temporomandibular joint dysfunction

syndrome

Facial pain

\begin{abstract}
A B S T R A C T
Introduction: Recently, some published studies show there is a multifactorial origin for Temporomandibular Disorders, but the dental occlusion's contribution to the development of Temporomandibular Disorders, and how it may influence the adaptive capacity of the Stomatognathic system, it's still unclear. The aim of this study is to evaluate the correlation between the Occlusion Time and Temporomandibular Disorders. Methods: A total of 54 patients were enrolled in the study (24 males and 30 females, mean age $27.94 \pm 8.21$ years). The TMD group ( 8 males and 10 females) consisted of subjects who presented with at least 1 of the following signs of Temporomandibular Disorders: Temporomandibular Joint sounds (clicking or crepitation), Temporomandibular Joint locking episodes, limited mandibular opening, painful limitation of mandibular movements, pain to palpation of the Temporomandibular Joint or of the masticatory muscles. The control group (16 males and 20 females) presented as free from Temporomandibular Disorders. The T-Scan III computerized occlusal analysis system was to record the subjects' Occlusion. Times during eight mandibular opening-closing movements. Results: The two-ways ANOVA test analyzed the variations for group and sex, showing that the TMD group mean Occlusion Time $(0.64 \pm 0.21 \mathrm{~s})$ was statistically significantly longer than the control group mean Occlusion Time $(0.45 \pm 0.17 s)(p<0.001)$. Significant differences were also found for gender where the mean OT of female subjects was longer than males one with statistical significance ( $p$-value $<0.01)$. Conclusions: The computerized analysis of the Occlusion Time in patients affected by TMJ problems has to be carefully considered as adjunctive instrumental device.
\end{abstract}

(C) 2014 Published by Elsevier Ltd.

\section{Introduction}

Temporomandibular Disorders (TMD) is a collective term which includes a group of clinical conditions affecting the stomatognathic system, in particular the muscles of mastication and the Temporomandibular joints (TMJ), which is characterized by a group of commonly reported symptoms: fairly localized pain, limited or asymmetric mandibular movements and TMJ noises (crepitations or clickings) (Okeson, 1997).

Because of the different methods used to assess TMD symptoms, which are often subjective, literature reports that the world-wide prevalence of TMD varies between $7 \%$ and $84 \%$ of the population within an age range of 3-74 years (Luther, 2007). Mostly it seems to afflict $10 \%$ of the population over 18 years of

* Corresponding author. Address: "Centro Medico Polispecialistico Baldini Srl" Via S. Orsola, n. 5 Bergamo, Postcode 24122, Italy. Tel.: +39 035 271935; fax: +39 035694280.

E-mail addresses: studiomedicobaldini@gmail.com (A. Baldini), notalex@ hotmail.it (A. Nota), paolacozza@alice.it (P. Cozza). age, where chronic pain is experienced in the Temporomandibular region (LeResche, 1997).

In fact, because of the variability of the symptoms of this cluster disorder, that potentially confirm a diagnosis of TMD, it has been quite problematic to isolate a primary etiology that predisposes the patients to suffer symptoms, leading researchers to advocate that TMD is of multifactorial etiology. Possible causes for TMD are Bruxism, mandibular muscle activity, facial growth, and also other systemic, postural, metabolic, structural, traumatic, psychological, social and behavioral influences, which have been identified as possible predisposing, initiating, and maintaining factors for TMD (Baldini et al., 2013a; De Boever and Carlsson, 1994).

For many years Dental Medicine had considered an unfavorable dental occlusion as a principal cause of the occurrence of TMD, because TMD appeared to be more frequently observed in patients with compromised or excessive vertical dimensions (De Boever et al., 2000).

However, later studies suggested TMD had a multifactorial origin such that dental occlusion was considered to be only one factor that may affect the adaptive capacity of the Stomatognathic 
system, which could lead to the development of TMD (McNamara, 1997).

Recent review articles (Luther, 2007; Türp and Schindler, 2012) underline that the evidence supporting a relationship between occlusion and TMD is still not voluminous, such that the authors suggested that the occlusion played a minor role in the development of the signs and symptoms Temporomandibular Disorders, noted that it would be important to establish evidence that a true cause and effect relationship exists between occlusion and TMD (Tecco et al., 2011).

The introduction of the Research Diagnostic Criteria for TMD (RDC/TMD) Axis I, provided standardized criteria for the diagnosis and classification of the different forms of TMD, categorizing them in three groups: Group I muscle disorders, Group II disc displacements and Group III other common joint disorders.

The RDC facilitated creating statistically better intra- and interexaminer reliability when clinicians were observing and reporting patients signs and symptoms and provided a common model for any examiner (Manfredini et al., 2006).

However, the frequently made, non-standardized diagnosis of TMD (absent of RDC/TMD international standards), combined with the low quality of some study designs, and, most importantly, the imprecise and subjective evaluation methods used in these studies to assess various aspects of the dental occlusion, have reduced the trustworthiness of these previous studies results (Baldini et al., 2012a).

The occlusal evaluation, in many studies that suggest occlusion is a minimal component in TMD etiology, have been routinely carried out with subjective methods:

the visual analysis of static occlusal contacts using articulating paper or occlusal wax, the visual analysis of dynamic occlusal contacts during voluntary or manipulated mandibular movements, the visual intra-oral analysis of the occlusion and the measurements obtained using millimeter rulers (Baba et al., 2000). This type of visually-gauged clinical examination is difficult to standardize (Wang and Yin, 2012) and has a limited reliability and validity, thus its trustworthiness and accuracy for research purposes is poor, and brings into question the conclusions of these studies that are based upon weak results (Gazit et al., 1986; Carey et al., 2007).

Considering these remarks, in research studies, the use of technology has become fundamental and should be encouraged. The research method then employs a sensible approach that can standardize measurements for comparisons of subjects, and provide measurable guidelines for improving conditions seen in daily practice (Manfredini et al., 2012; Baldini et al., 2013b).

Over the past 30 years, the evolution of diagnostic instrumentation, together with the clinical experience of practitioners, has given dental professionals a digital instrumental support, that improve the quality of information used in the diagnostic analysis (Baldini et al., 2012b, 2009). In 1987 Maness et al. (1987) reported the development of the first computerized system designed for occlusal analysis (T-Scan I, Tekscan, Inc. Boston, MA, USA). Notwithstanding its early problems (Harvey et al., 1992), its hardware and software evolution over the past 30 years, and improvements made to this technology which are included in the T-Scan III version (T-Scan III, Tekscan Inc. Boston, MA, USA with T-Scan HD sensor), ensured it is precise and reliable (Koos et al., 2010; Hirano et al., 2002).

The T-Scan III system allows for the recording the Occlusion Time (OT), which is a dynamic parameter defined as the elapsed time from initial tooth contact until maximum intercuspation is reached in centric occlusion (Kerstein and Grundset, 2001). It is directly related with each subject's occlusal contact pattern (Wang and Yin, 2012), thus some studies have considered this dynamic parameter as capable of describing a subjects' occlusion (Cheng et al., 2012; Sierpinska et al., 2008).
The aim of this study is to evaluate if there exists a correlation between Occlusion Time and Temporomandibular Disorders.

\section{Materials and Methods}

\subsection{Subjects}

A total of 54 Italian Caucasian patients were enrolled in the study (24 males and 30 females, mean age $27.94 \pm 8.21$ years) at the University of Rome Tor Vergata.

The ethical committee of the institute approved the protocol where all of the volunteers signed an informed consent form prior to their participation. An oral and anamnestic examination and TMJ screening of the subjects, was performed to seek out dental occlusion abnormalities or TMD present in the subjects. All the volunteers had to meet the following inclusion criteria: good general health according to a medical history, complete permanent dentition except for the third molars, absence of periodontal disease, absence of cast restorations and extensive occlusal restoration (less than 3 teeth with onlay restorations), occlusal Angle Class I or II (bilaterally assessed), absence of previous orthodontic therapy, absence of crossbite, absence of previous facial or mandibular traumas, good compliance with oral hygiene, absence of neurologic disturbances, absence of orthognathic surgery.

The enrolled subjects were divided in two groups: the TMD group ( 8 males and 10 females) affected by unilateral, long lasting, TMD muscle pain diagnosis (group Ia or Ib), disk displacements with reduction (group IIa), according to the RDC/TMD, axis I TMD and with presence of at least 1 of the following signs: TMJ sounds (clicking or crepitation), TMJ locking episodes, limited mandibular opening, painful limitation of mandibular movements, pain to palpation of the TMJ or of the masticatory muscles.

In particular, 8 subjects were affected by mild TMD and 10 subject by moderate TMD; 10 subjects were diagnosed for TMD group Ia, 5 subjects for TMD group Ib, 3 subject for TMD group Ila.

The control group (16 males and 20 females) subjects were healthy and free from all of the TMD symptoms described above in TMD group.

All subjects were unaware of the aims and/or design of the study.

\subsection{Equipment, procedure and statistical analysis}

The T-Scan III is a sophisticated computerized occlusal analysis system, that illustrates both static and dynamic occlusion. It can be used to analyze tooth contact patterns that occur within every mandibular movement (centric relation, centric occlusion, lateral excursions and protrusion), which can all be recorded in various mandibular postures, and body postures, as well. Therefore, the T-Scan system is much more than a simple evolution of an occlusal wax wafer, or a strip of articulating paper. Waxes and papers can visualize the occlusal contacts to the clinician, but do not show the occlusal force relationships between opposing occlusal contacts. Nor can they describe the order of sequential contact timing, nor how long each contact is maintained.

The T-Scan III device (Tek-Scan Inc., Boston, MA, USA) is approved as a "medical index center - scanning sensor system. It has multiple levels of recording sensitivity that can be adjusted at will, to match the recording level within the recording sensor, to the occlusal force exerted by the patient. The hardware comprised of a recording handle (which holds the recording sensor) that is connected to the computer through a USB port. Held securely within the recording handle is a "sensor support", which keeps the $100 \mu \mathrm{m}$ thick recording sensor properly extended flat 
within the mouth, during all intraoral recording procedures (Koos et al., 2012).

When a force is exerted on the sensor, occurs a voltage drop on its conduction paths that is immediately recorded by the software upon each tooth contact, and is then displayed for clinical analysis.

The T-Scan III computerized occlusal analysis system was used to record the subjects' Occlusion Time during 8 mandibular opening-closing movements. The patients sat upright in the dental chair with their head on the headrest and mandible parallel to the horizontal Frankfurt plane.

The Occlusion Times (OT) were recorded by placing the sensor between the dental arches, and asking the patient to perform 8 mandibular opening-closing movements without clenching, in and out of complete intercuspation (Kerstein and Grundset, 2001) with a velocity of about 1 movement per second. A single new sensor was used for each subject and all recordings were made by the same operator between 10:00 AM and 1:00 PM hours, in order to exclude any inter-rater and/or diurnal variability. Thus, an average value of the time between the first dental contact and the reaching of the habitual intercuspal contact position was obtained for each subject of both subject groups distribution of the Occlusion Times recorded, were summarized as means and SDs (standard deviation), according to the group belonging.

Repeated recording of another set of Occlusion Times were obtained on 28 subjects to evaluate the method error of the recordings using the Dahlberg's formula in order to verify the hypothesis of a non-significant difference between the mean values of the two groups' Occlusion Times.

In particular, the Occlusion Time of each subject was recorded twice, in the same conditions used for the TMD and control group recordings.

A two-ways ANOVA test was performed with Minitab 15 software.

Results were considered to be significant at the $5 \%$ critical level $(p<0.05)$.

\section{Results}

A total of 54 subjects included in the study underwent the recording of their Occlusion Times during 8 mandibular openingclosing movements.

The OT values were recorded and a mean OT value was calculated for each subject, where the TMD group mean OT $(0.64 \pm 0.21 \mathrm{~s})$ was about $0.18 \mathrm{~s}$ longer than the control group mean OT $(0.45 \pm 0.17 \mathrm{~s})$ (Table 1$)$. All the data were normally distributed according to Shapiro-Wilk's test. The method error calculated using the Dahlberg's formula was $0.015 \mathrm{~s}$. The variances were homoscedastic.

Analyzing the data of this study, sensitivity and specificity of this test are respectively $55 \%$ and $72 \%$.

The two-ways ANOVA test analyzed the variations for group and sex, showing that the TMD group mean Occlusion Time was statistically significantly longer than the control group mean Occlusion Time $(p=0.0005)$.

Table 1

Descriptive statistics.

\begin{tabular}{llll}
\hline Group & Mean $(\mathrm{s})$ & SD & Difference \\
\hline TMD & 0.6374 & 0.2143 & 0.1766 \\
Control & 0.4608 & 0.1636 & \\
TMD (males) & 0.4843 & 0.1307 & 0.2757 \\
TMD (females) & 0.7600 & 0.1895 & \\
Control (males) & 0.4196 & 0.1264 & 0.0742 \\
Control (females) & 0.4938 & 0.1887 & \\
\hline
\end{tabular}

Significant differences were also found for gender where the mean OT of female subjects was longer than males one with statistical significance $(p$-value $=0.0025)$.

\section{Discussion}

The analysis of the relationship between dental occlusion and TMD is not well defined in published studies, and is characterized by a disparity of results likely resultant from the low reproducibility of the different assessment methods used in the analysis of the subject's dental occlusion.

In the presented study, two young-adult population samples that were similar in age and gender were selected, a single operator, and a fixed diurnal time for the recordings of the Occlusion Times were established, to attempt to reduce the variability of the results. Moreover, eight Occlusion Times were recorded for each subject, and a mean value was calculated in order to approach a representative OT value per subject. The accuracy of the T-Scan system recording has been shown to be not altered by repeated measures, which was confirmed by other sensor and system recording accuracy studies (Koos et al., 2010; Hirano et al., 2002). The T-Scan system has been shown to repetitively record timed occlusal contacts quantitatively and dynamically, during a continuous mandibular movement (Kerstein et al., 1997; Wang and Yin, 2012).

The Occlusion Time is defined as the time between the first occlusal contact and the reaching of the complete habitual intercuspation (Kerstein and Grundset, 2001). The OT length can be correlated to the existence of premature occlusal contacts, and occlusal instability when closing into complete intercuspation. In this study, subjects with TMD showed significant differences from the control group who presented without TMD symptoms. In fact, the subjects affected by TMD had Occlusion Times about $0.18 \mathrm{~s}$ longer than the non-TMD subjects.

Of note was that female subjects seem to have higher Occlusion Times than males and this gender difference increase if they are affected by TMD, probably because women are more prone to be affected by TMD.

The reported results suggest that there is a deterioration in occlusal stability in subjects with TMD. In fact, premature contacts can result in condylar displacement, which may cause friction and increased intra-articular pressure on the Temporomandibular Joint, contributing to alteration of the disc position on the condyle, possibly leading to the onset of Temporomandibular Disorders, that can have negative effects on muscle activity levels (Kerstein and Radke, 2012).

The results of this study are in agreement with the findings of Wang and Yin (2012) that found higher Occlusion Times were present in subjects with Temporomandibular Disorders. However, the Wang and Yin study reported mean values differed from those of this study, both in the non-TMD subjects $(0.69 \mathrm{~s})$ and in Temporomandibular Disorders subjects $(2.05 \mathrm{~s})$. Surprisingly the Wang and Yin mean OT values in healthy subjects were similar to the mean OT values reported in this study, when recorded in subjects affected by TMD.

Contrarily, in other studies investigating the OT recordings in non-TMD subjects (Cheng et al., 2012; Sierpinska et al., 2008), their reported mean OT values appeared to be closer to the results of this study. Whereas, in subjects affected by Temporomandibular Disorders, this study's results appear very different from those of Wang and Yin, but unfortunately the scientific literature lacks studies about the OT in subjects affected by TMD. It seems difficult to state the reason for these differences between the two studies, where the methods used were largely similar. One possible explanation for the reported differences could be that the subjects in Wang and Yin's study were suffering from more severe TMD (their sample included TMD patients with at least 2 signs of TMD). 
Analyzing the data of this study, sensitivity and specificity of this test are respectively $55 \%$ and $72 \%$. However the sample is too small to obtain precise information about the use of the analysis of the Occlusion Time for diagnostic purposes. This result could also be related to the high variability of the clinical presentation of TMD notwithstanding in the design of the study, there was the attempt to create a quite homogeneous sample.

The results of this study could be an interesting starting point to set up an analysis of the Occlusion Time among samples of subjects affected by TMD with highly similar clinical presentation.

Within the limitations of this study's design, the computerized analysis of dental occlusion in patients affected by TMJ problems has to be carefully considered as adjunctive instrumental device. Further studies should be conducted to show a better matching between clinical TMJ signs and Occlusion Time. Moreover, females seem to have a higher Occlusion Time than males especially if affected by TMD.

\section{Conflict of interest}

The authors declare that they have no conflict interest.

\section{References}

Baba K, Tsukiyama Y, Clark GT. Reliability, validity, and utility of various occlusal measurement methods and techniques. J Prosthet Dent 2000;83(1):83-9.

Baldini A, Beraldi A, Nanussi A. The clinical importance of computerized evaluation of occlusion. Dental Cadmos 2009;77(4):47-54.

Baldini A, Beraldi A, Nota A, Danelon F, Ballanti F, Longoni S. Gnathological postural treatment in a professional basketball player: a case report and an overview of the role of dental occlusion on performance. Ann Stomatol (Roma) 2012a:3(2):51-8.

Baldini A, Tecco S, Cioffi D, Rinaldi A, Longoni S. Gnathological postural treatment in an air force pilot. Aviat Space Environ Med 2012b;83(5):522-6.

Baldini A, Nota A, Cravino G, Cioffi C, Rinaldi A, Cozza P. Influence of vision and dental occlusion on body posture in pilots. Aviat Space Environ Med 2013a;84(8):823-7.

Baldini A, Nota A, Tripodi D, Longoni S, Cozza P. Evaluation of the correlation between dental occlusion and posture using a force platform. Clinics 2013b;68(1):45-9.

Carey JP, Craig M, Kerstein RB, Radke J. Determining a relationship between applied occlusal load and articulating paper mark area. Open Dent J 2007;1:1-7.

Cheng HJ, Geng Y, Zhang FQ. The evaluation of intercuspal occlusion of healthy people with T-Scan II system. Shanghai Kou Qiang Yi Xue 2012;21:62-5.

De Boever JA, Carlsson GE. Etiology and differential diagnosis. In: Zarb GA, Carlsson GE, Sessle BE, Mohl ND. Temporomandibular joint and masticatory muscle disorders. 2nd ed. Copenaghen: Munksgaard - Mosby; 1994. p. 171-87.

De Boever JA, Carlsson GE, Klineberg IJ. Need for occlusal therapy and prosthodontic treatment in the management of Temporomandibular Disorders. Part II. Tooth loss and prosthodontic treatment. J Oral Rehabil 2000;27:647-59.

Gazit E, Fitzig S, Lieberman MA. Reproducibility of occlusal marking techniques. J Prosthet Dent 1986;55:505-9.

Harvey WL, Osborne JW, Hatch RA. A preliminary test of the replicability of a computerized occlusal analysis system. J Prosthet Dent 1992;67:697-700.

Hirano S, Okuma K, Hayakawa I. In vitro study on accuracy and repeatability of the T-Scan II system. Kokubyo Gakkai Zasshi 2002;69:194-201.

Kerstein RB, Grundset K. Obtaining bilateral simultaneous occlusal contacts with computer analyzed and guided occlusal adjustments. Quintessence Int 2001;32:7-18

Kerstein RB, Radke J. Masseter and temporalis excursive hyperactivity decreased by measured anterior guidance development. Cranio 2012;30:243-54.

Kerstein RB, Chapman R, Klein M. A comparison of ICAGD (Immediate complete Anterior Guidance Development) to "mock ICAGD" for symptom reductions in chronic myofascial pain dysfunction patients. J Craniomandib Pract 1997;15(1):21-37.

Koos B, Godt A, Schille C, Göz G. Precision of an instrumentation-based method of analyzing occlusion and its resulting distribution of forces in the dental arch. J Orofac Orthop 2010;71:403-10.

Koos B, Höller J, Schille C, Godt A. Time-dependent analysis and representation of force distribution and occlusion contact in the masticatory cycle. J Orofac Orthop 2012;73:204-14.

LeResche L. Epidemiology of Temporomandibular Disorders: implications for the investigation of etiologic factors. Crit Rev Oral Biol Med 1997;8:291-305.

Luther F. TMD and occlusion part II. Damned if we don't? Functional occlusal problems: TMD epidemiology in a wider context. Br Dent J 2007;202(E3):38-9.
Maness WL, Benjamin M, Podoloff R, Bobick A, Golden RF. Computerized occlusal analysis: a new technology. Quintessence Int 1987;18:287-92.

Manfredini D, Chiappe G, Bosco M. Research diagnostic criteria for Temporomandibular Disorders (RDC/TMD) axis I diagnoses in an Italian patient population. J Oral Rehabil 2006;33:551-8.

Manfredini D, Castroflorio T, Perinetti G, Guarda-Nardini L. Dental occlusion, body posture and Temporomandibular Disorders: where we are now and where we are heading for. J Oral Rehabil 2012;39:463-71.

McNamara JA. Orthodontic treatment and Temporomandibular Disorders. Oral Surg Oral Med Oral Pathol Oral Radiol Endod 1997;83:107-17.

Okeson JP. Current terminology and diagnostic classification schemes. Oral Surg Oral Med Oral Pathol Oral Radiol Endod 1997;83:61-4.

Sierpinska T, Golebiewska M, Lapuc M. The effect of mastication on occlusal parameters in healthy volunteers. Adv Med Sci 2008;53:316-20.

Tecco S, Crincoli V, Di Bisceglie B, Saccucci M, Macrì M, Polimeni A, et al. Signs and symptoms of Temporomandibular joint disorders in Caucasian children and adolescents. Cranio 2011;29:71-9.

Türp JC, Schindler H. The dental occlusion as a suspected cause for TMDs: epidemiological and etiological considerations. J Oral Rehabil 2012;39:502-12.

Wang C, Yin X. Occlusal risk factors associated with Temporomandibular Disorders in young adults with normal occlusions. Oral Surg Oral Med Oral Pathol Ora Radiol 2012;114:419-23.

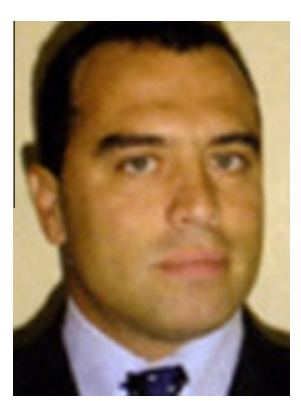

Alberto Baldini obtained his degree in 1989 at the University of Milano. He studied at the post-graduated School of Orthodontics at the University of Milano. He achieved a Ph.D. in Experimental Periodontology at the University of Milano-Bicocca. He is currently attending to his second Ph.D. in Materials for Health Environment and Energy at the University of Rome Tor Vergata with Prof. Paola Cozza. Expert in orthodontics and gnathology, author of articles on journals with Impact Factor.

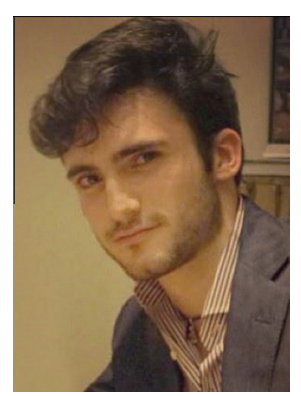

Alessandro Nota graduated in Dentistry cum Laude in 2011 at the University of Milano-Bicocca. His thesis was on the use of the force platform in the analysis of the correlation between dental occlusion and posture. In 2013 he obtained an MSc in Orthodontics at the University of Rome Tor Vergata with Prof. Paola Cozza. In the late 2013 he started his PhD in Materials for Health Environment and Energy applied to dentistry and orthognathology at the University of Rome Tor Vergata. Author of articles on scientific journals with Impact Factor in these fields.

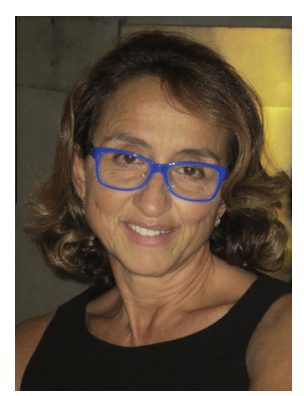

Paola Cozza is presently Full Professor, Dean of the Dental School and head of the Postgraduate Program in Orthodontics at the University of Rome Tor Vergata, Italy. She graduated cum laude in Medicine (MD), and she graduated in Dentistry (DDS) at the University of Rome, La Sapienza, Italy, in 1988. In 1991 she's got the degree of specialist in Orthodontics and since then she has been a clinical istructor in the program in Orthodontics. She is the author of over 100 scientific articles in journals with Impact Factor, in the American Journal of Orthodontics and Dentofacial Orthopedics, in the Angle Orthodontist, in the European Journal of Orthodontics and in European Journal of pediatric dentistry. She has written or otherwise contributed to 18 textbooks. She sees patients in the faculty associates practice in the Department of Orthodontics at the University of Rome "Tor Vergata". Prof. Cozza is a member of the Italian Society of Orthodontics (S.I.D.O.), European Orthodontic Society (E.O.S.), and World Federation of Orthodontists (W.F.O.). She is Incoming President of S.I.D.O. She is a member of the panel of referee of many national and international journals. 